\title{
Tuberculosis infection control in a South African rural regional hospital emergency centre: Prioritisation for patients and healthcare workers
}

\author{
S A Scott, ${ }^{1}$ BMBS, BSc, BMedSci, DMMC; N van Zyl Smit, ${ }^{2}$ MB ChB, FRCEM; \\ L S Jenkins, ${ }^{3}$ MB ChB, MFamMed, DA (SA), Dip (Obst) (SA), DHSM, FCFP (SA), PhD \\ ${ }^{1}$ NHS Thames Valley and Wessex Leadership Academy, Portsmouth, UK \\ ${ }^{2}$ Department of Family and Emergency Medicine, George Hospital, South Africa \\ ${ }^{3}$ Department of Family and Emergency Medicine, Faculty of Medicine and Health Sciences, Stellenbosch University, South Africa
}

Corresponding author: S A Scott (ss14742@my.bristol.ac.uk)

South Africa (SA) is in the midst of a tuberculosis (TB) epidemic and has one of the highest TB incidence rates globally. Despite increasing global commitment to eliminate TB, SA appears to be falling behind in this regard. This article examines key challenges to effective TB infection control from a rural regional hospital perspective. It uses the Eden District in Western Cape Province as an example to share lessons learnt. This quality-improvement project identifies four priorities for improving TB infection control in George Hospital and the Eden District: (i) prioritising TB infection control in local policy; (ii) improving the quality of TB screening in the emergency centre; (iii) increasing the number of TB patients followed up; and (iv) implementing TB infection control training for all staff. This project demonstrates the role of an emergency centre in TB screening, highlighting that this should not only be a priority for primary care, but also for secondary and tertiary care. Simple interventions, such as training of local healthcare workers in TB infection control and goodquality TB screening, can initiate a behavioural change. It also stresses the importance of good communication and co-ordination of care across primary and secondary care, ensuring that patients are not lost to follow-up. Local policy needs to reflect these straightforward interventions, empowering local healthcare workers and managers to increase responsibility and accountability for TB infection control.TB is preventable, and infection control needs to become a priority throughout SA primary, secondary and tertiary care. This project highlights that simple interventions, such as engaging local healthcare workers in a co-ordinated multisystem and multidisciplinary approach, could help to reduce the number of missing TB cases and bring SA's TB epidemic under control.

S Afr Med J 2019;109(8):555-558. DOI:10.7196/SAMJ.2019.v109i8.14039

Tuberculosis (TB) is the leading cause of death among patients with infectious diseases and accounts for 1 of 10 major causes of mortality globally. ${ }^{[1]}$ Since 2015, there has been a renewed global commitment regarding TB, with Sustainable Development Goal 3 (target 3.3) aiming to 'end the epidemic of TB, ${ }^{2]}$ and the 'End TB strategy', with the objective to 'reduce TB deaths by $95 \%$ and its incidence rate by $90 \%$ between 2015 and $2035^{\cdot[3]}$ Despite this, South Africa (SA) remains amid a TB epidemic and has one of the highest TB incidence rates in the world. ${ }^{[4]}$

Throughout SA, there is great concern for staff and patients with regard to TB transmission in public health facilities. ${ }^{[5-7]}$ It is estimated that healthcare workers are three times more likely to contract active TB and six times more likely to contract drug-resistant TB than the general population. ${ }^{[5,7]}$

Western Cape Province has a TB incidence rate of 643 per 100000 population, with an estimated 11812 missing TB cases, ${ }^{[8]}$ e.g. those not diagnosed and/or not initiated on treatment. In the Eden District of the Western Cape, TB is a significant burden, accounting for the leading cause of natural death. ${ }^{[9]}$ From a rural regional hospital perspective, this article identifies barriers to effective TB control in the Eden District. As TB is not limited to this district, the aim is to share the lessons learnt and to contribute to the widening evidence for urgent prioritisation of TB infection control in SA.
What this article adds

- TB infection control and screening should be a priority in primary, secondary and tertiary care in South Africa

- Training is essential for effective TB screening and infection control

- TB training can facilitate healthcare workers to become agents of behavioural change in TB infection control

- $\mathrm{TB}$ needs to become a priority in local policy

$\mathrm{TB}=$ tuberculosis.

\section{Highlighting the issues}

TB as a priority in policy

Healthcare policy is used to direct healthcare workers' behaviour and actions. Despite the large burden of TB in the Eden District, there is a disproportionate lack of local hospital and district policy on TB infection control. When TB infection control is referenced, especially in national policy, indicators are not necessarily correctly prioritised, e.g. in the Ideal Clinic Framework ${ }^{[10]}$ posters on cough etiquette are prioritised over TB infection control training, which is not directly mentioned. Targets are often general, such as in the national core standards, which require 'a programme for the prevention and control of respiratory infections to be in place. ${ }^{[11]}$ National targets, which are nonspecific, could be exacerbating the poor implementation and prioritisation of $\mathrm{TB}$ infection control in the local setting. 
It is hoped that the recent launch of the national strategy to 'find the missing TB cases' is the beginning of this prioritisation. ${ }^{[12]}$ Similar to the shortfalls identified in the Eden District, this strategy identified the need to 'optimise the quality of TB screening' and to 'improve the quality standards for recording, reporting and movement of patients between facilities. ${ }^{[12]}$ The latter emphasises the importance of increasing communication between the hospital and district healthcare facilities, which needs to be reflected in local hospital and district policy.

\section{Quality TB screening as a priority in secondary care}

$\mathrm{TB}$ is most commonly referred to as a primary healthcare problem. Due to the high morbidity and mortality resulting from TB, secondary and tertiary healthcare systems are increasingly burdened with these challenges. Of particular concern is the transmission of TB between patients and healthcare workers in hospitals. ${ }^{[5-7]}$ In $2015,20 \%$ of all cases of pulmonary TB in the George district were diagnosed at George Hospital, ${ }^{[13]}$ highlighting that TB is a diagnostic priority for hospitals and primary healthcare centres.

Between January and May 2018, 66\% of TB patients at George Hospital were diagnosed in the emergency centre. Using the Hospital Emergency Centre Triage Information System (HECTIS), $42 \%$ of patients were screened for TB between July and August 2018. However, it became evident that the nurses had received no training on how to use the TB screening tool and were therefore uninformed regarding the criteria for a TB 'screen-positive' patient and the appropriate actions required. Because of the poor quality of the screening process, the emergency centre had therefore screened very few patients effectively for TB.

Houben and Menzies ${ }^{[14]}$ noted that to achieve the World Health Organization (WHO) 2025 global TB targets, SA requires a combination of interventions, including expanded facility-based TB screening. Furthermore, SA's national infection control guidelines for $\mathrm{TB}^{[15]}$ recommend screening at all initial points of contact at healthcare facilities. The recent national strategy also highlights the priority of optimising the quality of facility-based TB screening. ${ }^{[12]}$

\section{Follow-up of TB patients}

In 2018, SA embarked on a national strategy to quantify and reduce the number of missing TB cases. ${ }^{[12]} \mathrm{A}$ significant proportion of these estimated cases are lost to follow-up, i.e. patients who have been diagnosed with TB but have not started treatment. From January to May 2018, only 57\% of patients with first evidence of TB at George Hospital started treatment. Therefore, there were 116 missing TB patients, without considering the possibility of further transmission from each of these patients (Western Cape provincial health data, TB care cascades, 30 November 2018 - unpublished data). A contributing factor was a distinct lack of communication between the hospital and the district. A number of patients with positive TB sputum samples collected in hospital were not initiated on treatment in their clinics. There was also minimal communication between the hospital infection control team and the sub-district TB co-ordinators. This high number of patients lost to follow-up is a waste of resources and may be contributing to the perpetuation of the TB epidemic within the district.

\section{TB infection control training}

Zinatsa et al. ${ }^{[16]}$ main recommendation to improve $\mathrm{TB}$ infection control in SA is education for healthcare workers and patients. Teaching increases awareness of the risk of TB transmission and TB infection control practices. Despite SA having one of the highest TB incidence rates in the world, there is no mandatory national training on TB infection control and screening for healthcare workers. Training is defined as an administrative control, which is at the top of the hierarchy of TB infection controls. ${ }^{[17]}$ Interestingly, the other controls, environmental and personal protective equipment, also require an element of training. TB infection control training is a low-cost intervention that focuses on prevention as opposed to cure.

\section{Intervention}

Four interventions were prioritised to improve TB infection control in George Hospital and the Eden District:

- Prioritising TB through the creation of a hospital TB infection control policy and TB risk assessment tool, the latter enabling the facility to monitor its progress in TB infection control.

- A TB screening procedure, whereby all emergency centre nurses were trained regarding the screening process. This involved dissecting the meaning of the screening questions, explaining how to ask open questions and clearly defining the criteria for a TB screen-positive patient and the appropriate actions.

- A follow-up pathway was created, which ensured that all subdistrict TB co-ordinators had access to George Hospital's positive $\mathrm{TB}$ sputum results. On receiving the positive samples, TB co-ordinators verified that treatment had been initiated and, if not, actively traced the patient. The objective was to reduce the number of missing TB cases and those lost to follow-up.

- A TB training programme for each emergency centre staff group was designed, including nurses, cleaners, security guards, clerks, doctors, porters and ambulance staff. Each staff group had a TB champion, who taught their respective group. The aim was to create a united approach to TB infection control, empowering all groups to protect patients and themselves. The training was interactive, relevant and simple and used patient videos from TB Proof, ${ }^{[18]}$ describing local healthcare workers' TB journeys. Our TB training resources can be accessed on the following link: https://drive. google.com/open?id=1FdsY4REwe-ejlO22_TMctF4xAhzW6YUq

\section{Discussion}

\section{TB as a priority in policy}

Prioritising TB in local policy has stimulated discussions, increased awareness and encouraged managerial and clinical commitment to reduce $\mathrm{TB}$ transmission. The TB risk assessment tool and infection control policy have accelerated accountability and responsibility for the hospital and healthcare workers, as they are now monitored on their ability to adhere to policy. Particularly useful was the outlining of the policy of the infection control committee's responsibilities. Our vision is for all facilities in the region to complete an annual TB risk assessment and have an individualised TB infection control policy. To encourage behavioural change in SA's healthcare workers, TB infection control has to become a priority in primary, secondary and tertiary care local policy.

\section{Quality TB screening as a priority in secondary care}

After the intervention, there was a significant increase in the number of patients screened for TB at the emergency centre (from $42 \%$ to $95 \%$ ) (Fig. 1). The positive TB screening rate remained similar, but in terms of actual patient numbers those who screened positive had tripled (Fig. 2). There were concerns that there would be a large increase in unnecessary sputum samples and the positive test rate would decrease; however, the percentage of samples that tested positive remained similar (14\% pre-intervention, $13 \%$ postintervention), and more TB cases were diagnosed. Despite the small sample size, it shows the potential of good-quality TB screening. 
Because TB was discussed at triage, regardless of the presenting complaint, more than double the number of known TB cases were identified (Fig. 3). Identifying TB earlier in the patient's journey enables nurses to identify those most at risk of TB transmission and

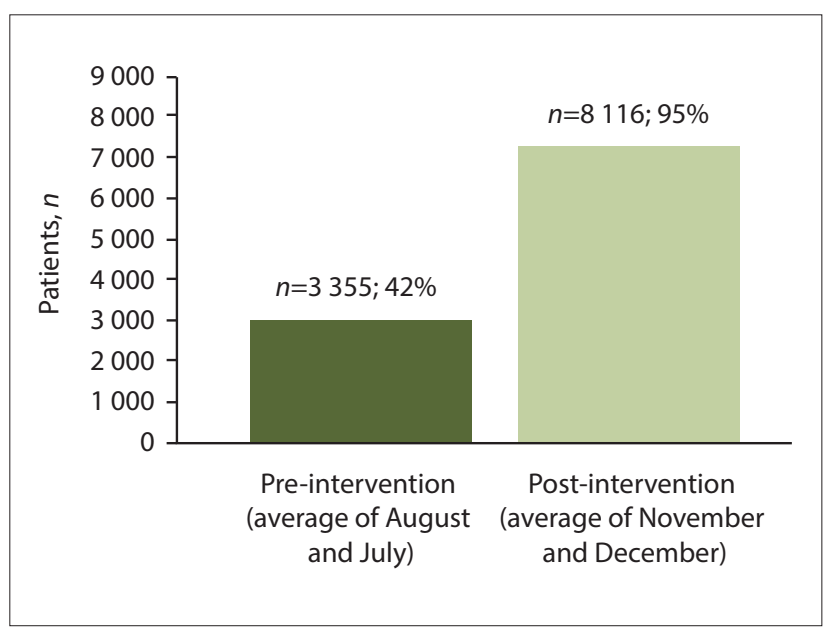

Fig. 1. Percentage and actual number of patients screened for tuberculosis before and after the intervention.

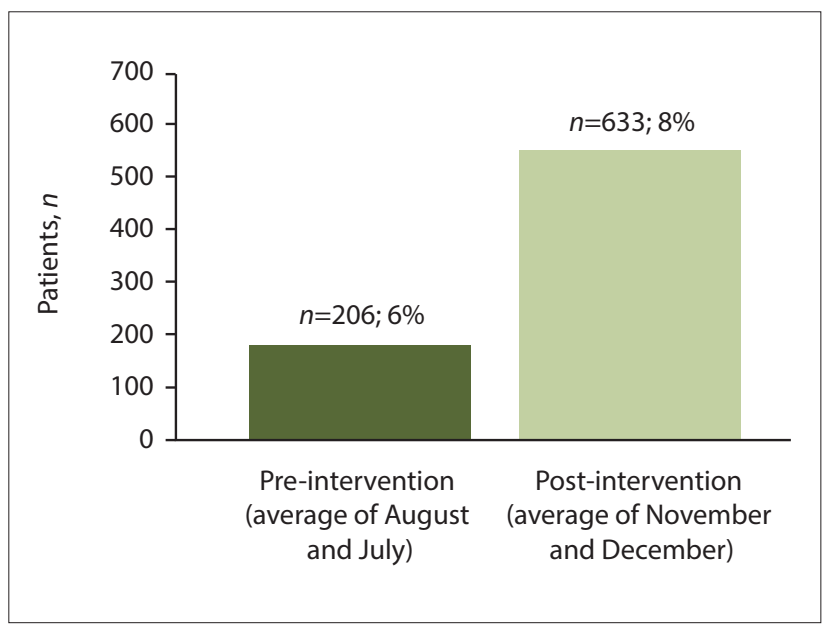

Fig. 2. Percentage and actual number of patients screened as tuberculosis positive before and after the intervention.

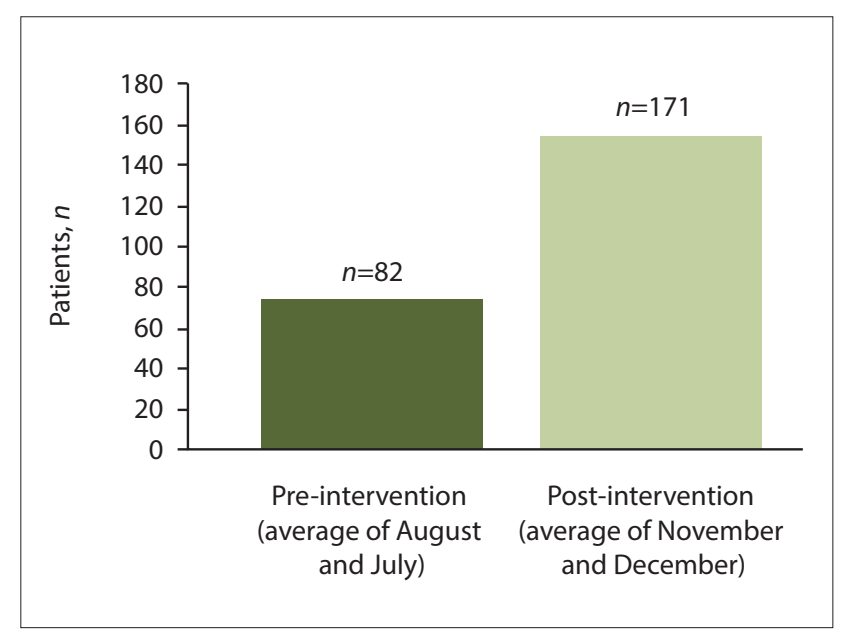

Fig. 3. Numbers of known tuberculosis patients identified at triage before and after the intervention. implement appropriate infection control practices. This intervention of ensuring good-quality screening has demonstrated the hospital's potential to contribute to reducing the transmission of $\mathrm{TB}$ in their facility and district.

\section{Follow-up of TB patients}

The national strategy prioritises improving quality standards for the movement of patients between facilities. ${ }^{\text {[12] }}$ It is too early to identify whether there is an improvement in the number of patients initiated on treatment with first evidence of TB at George Hospital. Nevertheless, the seed has been planted for improved communication between primary and secondary care, which has the potential to significantly reduce the number of missing $\mathrm{TB}$ cases. Improved communication could also benefit patient care across many other acute and chronic diseases. With regard to $\mathrm{TB}$, we recommend improved local platforms for discussion. Ideally, there should be a monthly meeting, where hospital and district staff could meet to discuss patients lost to follow-up, the number of patients who defaulted while receiving treatment, screening statistics and TB infection control risk assessment, training and policy. Without a united approach, patients will continue to receive suboptimal care and contribute to the perpetuation of the TB epidemic.

\section{TB infection control training}

Over the past decade, numerous studies have highlighted the poor implementation of $\mathrm{TB}$ infection control practices in healthcare facilities across SA. ${ }^{[6,16]}$ Providing training on TB infection control has increased the ability of our healthcare workforce to recognise the symptoms of TB and how to reduce its transmission at all points of the patient journey through our facility: from the clerk at registration asking if the patient has a cough, to the security guard in the waiting room showing the patient how to wear the mask correctly, to the triage nurse collecting sputum samples in a designated location.

As TB infection control is required of all hospital staff groups, we found it difficult to identify a healthcare worker responsible for this training. In an effort to create responsibility, criteria for training were added to the newly designed TB infection control risk assessment and policy. For SA to control its TB epidemic, responsibility and accountability need to be taken by managerial and clinical staff for TB infection control training. We recommend it as a priority for all primary, secondary and tertiary healthcare facilities in SA.

\section{Study limitations}

This quality-improvement project comprised a small sample size - the emergency centre of one regional hospital. The outcomes and lessons span only 5 months. This work is ongoing, and with a larger sample size and longer time period the outcomes and lessons may change. This study focused primarily on interventions in secondary care. To create an integrated approach, future studies need to perform qualityimprovement cycles across all levels of a healthcare system, creating a uniform method and responsibility for TB infection control. Since this was a quality-improvement project, ethical approval was not required.

\section{Conclusions}

This project is novel, as it highlights the role of a regional hospital in contributing to the multipronged approach to TB infection control. It identifies simple interventions, such as quality $\mathrm{TB}$ screening, $\mathrm{TB}$ infection control training and patient follow-up. It emphasises the importance of co-ordinated care between the hospital and community and the need for policies to go back to basics, reflecting 
simple interventions, engaging local healthcare workers in training and thus encouraging a behavioural change to TB infection control. $\mathrm{TB}$ is a preventable epidemic; it is not endemic and should be identified as a priority throughout SA primary, secondary and tertiary care.

\section{Declaration. None.}

Acknowledgements. The authors are grateful to all George Hospital staff for their support and their continued participation in the programme. Support from the CEO, Mr M Vonk, and the nursing lead, Ms E Sellars, is also acknowledged.

Author contributions. SAS conceptualised the original manuscript; SAS, NvZS and LSJ contributed to the subsequent drafts; and all authors added to and approved the final draft.

Funding. None.

Conflicts of interest. None.

1. World Health Organization. Global Tuberculosis Report. Geneva: WHO, 2018

2. World Health Organization. Sustainable development goals. https://www.who.int/sdg/targets/en/ (accessed 14 January 2019).

3. World Health Organization. Post 2015 End-TB Strategy. Global Strategy and Targets for Tuberculosis 3. World Health Organization. Post 2015 End-TB Strategy. Global
Prevention, Care and Control after 2015. Geneva: WHO, 2014.

Prevention, Care and Control after 2015. Geneva: WHO, 2014.
4. World Health Organization. Tuberculosis. Geneva: WHO, 2017.

4. World Health Organization. Tuberculosis. Geneva: WHO, 2017.
5. O'Donnell MR, Jarand J, Loveday M, et al. High incidence of hospital admissions with multidrugresistant and extensively drug-resistant tuberculosis among South African health care workers. Ann Intern Med 2010;153(8):516-522. https://doi.org/10.7326/0003-4819-153-8-201010190-00004
6. Sissolak D, Marais F, Mehtar S. TB infection prevention and control experiences of South African nurses a phenomenological study. BMC Public Health 2011;11:262. https://doi.org/10.1186/14712458-11-262

7. University Research Company (URC) South Africa. Tuberculosis in Healthcare Workers: Findings . University Research Company (URC) South Africa. Tuberculosis in Healthcare Workers: Findings
from South Africa. Cape Town: University Research Co. LLC and Desmond Tutu Tuberculosis Centre, 2013:2.

8. Krzywda R. Finding missing TB patients among key populations. 5th Southern Africa TB Conference, Durban, South Africa, 12 - 15 June 2018. https://www.usaid.gov/southern-africa-regional/speeches/ june-13-2018-5th-sa-tb-conference-tb-symposium- $\% \mathrm{E} 2 \% 80 \% 93$-finding-missing-tb-patientsamong-key (accessed 23 May 2019)

9. Eden District Management Team. Eden District Health Plan 2018/19 - 2020/21. http://www.health.gov. a/DHP/docs/DHP2018-21/Western_Cape/Eden_District_DHP_2018-19.pdf (accessed 15 January 2019).

10. National Department of Health. Ideal Clinic Definitions, Components and Checklists. Version 18. Pretoria: $\mathrm{NDoH}, 2018$.

11. National Department of Health. National Core Standards for Health Establishments in South Africa. Pretoria: NDoH, 2011.

12. National Department of Health. Finding missing TB patients workplan. South Africa. 2018. https:// tbsouthafrica.org.za/ (accessed 23 May 2019).

13. Casey H, Smith A, Parker L, Dipper M, Gould T. Pulmonary tuberculosis in a South African regional . Casey H, Smith A, Parker L, Dipper M, Gould T. Pulmonary tuberculosis in a South African regional
emergency centre: Can infection control be improved to lower the risk of nosocomial transmission? emergency centre: Can infection control be improved to lower the risk of

14. Houben RMGJ, Menzies NA. Feasibility of achieving the 2025 WHO global tuberculosis targets in South Africa, China, and India: A combined analysis of 11 mathematical models. Lancet Global Health 2016;4(11):e806-e815. https://doi.org/10.1016/S2214-109X(16)30199-1

15. National Department of Health. National Infection Prevention and Control Guidelines for TB, MDRTB and XDR-TB. Pretoria: NDoH, 2015.

16. Zinatsa F, Engelbrecht M, van Rensburg AJ, Kigozi G. Voices from the frontline: Barriers and strategies to improve tuberculosis infection control in primary health care facilities in South Africa. BMC Health Serv Res 2018;18(1):269. https://doi.org/10.1186/s12913-018-3083-0

17. World Health Organization. WHO Policy on TB Infection Control in Health-care Facilities, Congregate Settings and Households. Geneva: WHO, 2009.

18. What is TB Proof? http://www.tbproof.org/ (accessed 15 January 2019).

Accepted 27 May 2019. 